\title{
Electron Affinities of the DNA and RNA Bases
}

\author{
Steven S. Wesolowski, ${ }^{\dagger, \downarrow}$ Matthew L. Leininger, ${ }^{\S}$ Plamen N. Pentchev ${ }^{\dagger}, \perp$ and \\ Henry F. Schaefer III*,†
}

Center for Computational Quantum Chemistry, University of Georgia, Athens, Georgia 30602-2525, Sandia National Laboratory, MS 9217 P.O. Box 969, Livermore, California 94551-0969, and Center for Analytical Chemistry and Applied Spectroscopy, University of Plovdiv, Plovdiv, Bulgaria

Received October 30, 2000. Revised Manuscript Received February 28, 2001

\begin{abstract}
Adiabatic electron affinities (AEAs) for the DNA and RNA bases are predicted by using a range of density functionals with a double- $\zeta$ plus polarization plus diffuse $(\mathrm{DZP}++)$ basis set in an effort to bracket the true EAs. Although the AEAs exhibit moderate fluctuations with respect to the choice of functional, systematic trends show that the covalent uracil $(\mathrm{U})$ and thymine $(\mathrm{T})$ anions are bound by $0.05-0.25 \mathrm{eV}$ while the adenine (A) anion is clearly unbound. The computed AEAs for cytosine (C) and guanine (G) oscillate between small positive and negative values for the three most reliable functional combinations (BP86, B3LYP, and BLYP), and it remains unclear if either covalent anion is bound. AEAs with B3LYP/TZ2P++ single points are $0.19(\mathrm{U}), 0.16(\mathrm{~T}), 0.07(\mathrm{G}),-0.02(\mathrm{C})$, and $-0.17 \mathrm{eV}(\mathrm{A})$. Favorable comparisons are made to experimental estimates extrapolated from photoelectron spectra data for the complexes of the nucleobases with water. However, experimental values scaled from liquid-phase reduction potentials are shown to overestimate the AEAs by as much as $1.5 \mathrm{eV}$. Because the uracil and thymine covalent EAs are in energy ranges near those of their dipole-bound counterparts, preparation and precise experimental measurement of the thermodynamically stable covalent anions may prove challenging.
\end{abstract}

\section{Introduction}

The initial step of high-energy radiation damage to DNA and RNA is suspected to be the formation of transient charged radicals within the strand. Specifically, electron trapping within the purine and pyrimidine nucleobase sites is believed to play a key role in DNA damage and repair. ${ }^{1-4}$ While these mechanisms are inherently complex, the ease of reduction of each nucleobase (guanine $\mathrm{G}$, adenine $\mathrm{A}$, cytosine $\mathrm{C}$, thymine $\mathrm{T}$, and uracil $\mathrm{U}$ ) is directly correlated with its adiabatic electron affinity (AEA). Thus, an accurate knowledge of these well-defined thermodynamic properties may provide insight into likely locations of initial nucleotide modifications which precede the cascade of reactions leading to mutations. Additionally, further understanding of the novel (and often controversial) notion of charge transfer and electron flow along the $\pi$ stack of nucleobases also relies upon a knowledge of the response of these moeities to excess negative charge. ${ }^{5-12}$

\footnotetext{
University of Georgia.

Current address: Department of Chemistry, Yale University, New Haven, CT 06520-8107.

$\S$ Sandia National Laboratory.

${ }^{\perp}$ University of Plovdiv.

(1) Steenken, S.; Telo, J. P.; Novais, H. M.; Candeias, L. P. J. Am. Chem. Soc. 1992, 114, 4701.

(2) Colson, A. O.; Sevilla, M. D. Int. J. Radiat. Biol. 1995, 67, 627.

(3) Desfrançois, C.; Abdoul-Carime, H.; Schermann, J. P. J. Chem. Phys. 1996, 104, 7792.

(4) Huels, M. A.; Hahndorf, I.; Illenberger, E.; Sanche, L. J. Chem. Phys. 1998, 108, 1309.

(5) Hall, D. B.; Holmlin, R. E.; Barton, J. K. Nature 1996, 382, 731.

(6) Warman, J. M.; de Haas, M. P.; Rupprecht, M. Chem. Phys. Lett. 1996, $249,319$.

(7) Poryadarshy, S.; Risser, S. M.; Beratan, D. N. J. Phys. Chem. 1996, 100,17678

(8) Taubes, G. Science 1997, 275, 1420

(9) Lewis, F. D.; Wu, T.; Zhang, Y.; Letzinger, R. L.; Greenfield, S. R.; Wasielewski, M. R. Science 1997, 277, 673.
}

Despite many experimental and theoretical efforts, precise values for the adiabatic electron affinities of the nucleobases have remained elusive (see refs 13 and 14 for recent overviews). While there have been no direct measurements of the nucleobase AEAs, experimentally based estimates were first derived from those of pyrimidine and purine by using substitution and replacement rules. ${ }^{15}$ Each anion was predicted to be strongly bound with an AEA of at least $0.60 \mathrm{eV}$. The guanine anion was reported to be particularly stable with an electron affinity of $1.05 \mathrm{eV}$. Later studies by Chen and Chen utilized the reversible reduction potentials of the nucleobases in an aprotic solvent, and the AEAs were estimated by using scaling factors based on the known gas-phase EAs for acridine and anthracene. ${ }^{16}$ These results also suggested that all the nucleobases have substantial AEAs with values ranging from $0.56 \mathrm{eV}$ for cytosine to $1.51 \mathrm{eV}$ for guanine. The relative AEAs from these studies can be summarized as

$$
\mathrm{C}<\mathrm{T}<\mathrm{U}<\mathrm{A} \ll \mathrm{G}
$$

In direct contrast, most ab initio computations have predicted negative nucleobase valence adiabatic electron affinities for several of the DNA bases (see Table 1). For example, second-

\footnotetext{
(10) Dandliker, P. J.; Holmlin, R. E.; Barton, J. K. Science 1997, 275, 1465 .

(11) Stemp, E. D. A.; Arkin, M. R.; Barton, J. K. J. Am. Chem. Soc. 1997, 119, 2921.

(12) Diederichsen, U. Angew. Chem. 1997, 109, 2411.

(13) Hendricks, J. H.; Lyapustina, S. A.; de Clercq, H. L.; Bowen, K. H. J. Chem. Phys. 1998, 108, 8.

(14) Wetmore, S. D.; Boyd, R. J.; Eriksson, L. A. Chem. Phys. Lett. 2000, 322, 129.

(15) Chen, E. C. M.; Chen, E. S. D.; Wentworth, W. E. Biochem. Biophys. Res. Commun. 1990, 171, 97.

(16) Wiley, J. R.; Robinson, J. M.; Ehdaie, S.; Chen, E. C. M.; Chen, E. S. D.; Wentworth, W. E. Biochem. Biophys. Res. Commun. 1991, 180, 841
} 
Table 1. Previously Reported Adiabatic Electron Affinities (in eV) of the DNA and RNA Bases ${ }^{f}$

\begin{tabular}{lrrrrrrr}
\hline & B3LYP $^{a}$ & B3LYP $^{b}$ & B3LYP $^{c}$ & MP2 $^{d}$ & PMP2 $^{a}$ & Koopmans $^{d}$ & AM1 $^{e}$ \\
\hline uracil & 0.18 & 0.07 & 0.14 & -0.25 & -0.12 & -0.7 & 0.87 \\
thymine & 0.14 & & 0.08 & -0.30 & -0.17 & 0.3 & 0.90 \\
cytosine & -0.06 & & -0.12 & -0.46 & -0.27 & 0.2 & 0.82 \\
guanine & -0.27 & & -0.38 & -0.75 & -0.61 & -0.3 & 1.48 \\
adenine & -0.40 & & -0.48 & -1.19 & -0.73 & 0.4 & 1.06
\end{tabular}

${ }^{a} 6-311+\mathrm{G}(2 \mathrm{df}, \mathrm{p})$ basis set used. See ref $14 .^{b} 6-311++\mathrm{G}$ basis set used. Zero-point correction not included. See ref 20. ${ }^{c}$ TZVP basis set used. See ref 28. ${ }^{d}$ Scaled Koopmans energies. See ref 2. ${ }^{e}$ Reference 51. ${ }^{f}$ All computed values are zero-point corrected unless otherwise noted.

order Møller-Plesset perturbation theory (MP2) with a modest basis set including diffuse functions yields AEAs ranging from -1.19 to $-0.25 \mathrm{eV}$ for the five molecules. Unlike the experiments of Chen and co-workers, ${ }^{15,16}$ the MP2 results also predict the AEAs of the pyrimidines $(\mathrm{C}, \mathrm{T}$, and $\mathrm{U})$ to be much greater than those of the purines $(\mathrm{G}$ and $\mathrm{A})$

$$
\mathrm{A}<\mathrm{G} \ll \mathrm{C}<\mathrm{T}<\mathrm{U}
$$

In 1998, Bowen and co-workers assessed the state of the problem noting, "Despite voluminous evidence that nucleobase anions exist in both solutions and the solid state, none of these [previous] calculations found stable anions of nucleic acid bases in isolation, i.e., the [adiabatic] EAs of their neutrals had negative values."13

Recently, more sophisticated experiments and theoretical computations have simultaneously shed new light and cast longer shadows upon this problem. In the early 90s, Adamowicz and co-workers first predicted the existence of a so-called "dipole-bound" anion for uracil. ${ }^{17}$ Noting the substantial dipole moment of uracil, they proposed that an additional electron could establish itself in a very diffuse molecular orbital and weakly bind via dipole interactions rather than fill the lowest unoccupied molecular orbital as in conventional "covalent" anions. Their MP2 prediction of a very small, but positive, dipole-bound electron affinity (DBEA) for uracil $(0.086 \mathrm{eV})$ has now been verified experimentally. ${ }^{3,18}$ Indeed, both Bowen et al. ${ }^{18}$ and Schermann et al. ${ }^{3}$ independently observed characteristics suggestive of a dipole-bound anion in negative photoelectron spectroscopy (PES) and Rydberg electron transfer (RET) spectroscopy studies, respectively. However, no conclusive sign of a covalent uracil anion was observed in either of these pioneering experiments.

Given the existence of a dipole-bound uracil anion in the gas phase and conclusive electron spin resonance evidence that the DNA base anions are conventional (covalent) anions in solution, ${ }^{19}$ Bowen and co-workers performed PES studies on the naked and microsolvated uracil anion to assess the point at which the uracil anion converts from dipole bound to covalently bound. ${ }^{13}$ Interestingly, the crossover is immediate, as attachment of a single water molecule to the uracil anion exclusively yields a broad photoelectron spectrum indicative of a covalently bound anion. However, when water is replaced by a xenon atom as the solvating agent, both dipole-bound and covalent anion complexes are observed. Figure 1 illustrates the spectral signature of each type of anion. The sharp structure in the photoelectron spectrum for $\mathrm{U}^{-}$also suggests that the observed vertical transition is likely very near that of the adiabatic dipole-

(17) Oyler, N. A.; Adamowicz, L. J. Phys. Chem. 1993, 97, 11122.

(18) Hendricks, J. H.; Lyapustina, S. A.; de Clercq, H. L.; Snodgrass, J. T.; Bowen, K. H. J. Chem. Phys. 1996, 104, 7788.

(19) See for example: Close, D. M. Radiat. Res. 1993, 135 and references therein.

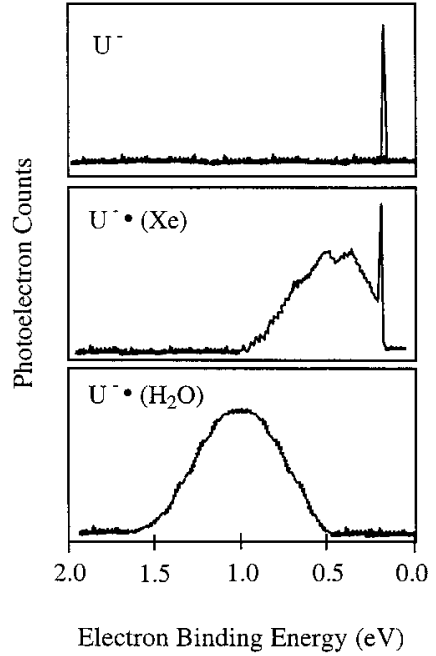

Figure 1. Schematic reproductions of the uracil anion photoelectron spectra for $\mathrm{U}^{-}, \mathrm{U}^{-} \cdot(\mathrm{Xe})$, and $\mathrm{U}^{-} \cdot\left(\mathrm{H}_{2} \mathrm{O}\right)$ from ref 13 . The sharp peak for free $\mathrm{U}^{-}$is characteristic of a dipole-bound anion, while the broad peak for $\mathrm{U}^{-} \cdot\left(\mathrm{H}_{2} \mathrm{O}\right)$ is indicative of a covalently bound anion. The uracil anion complex with xenon exhibits both types of anions.

bound transition. Indeed, the vertical detachment energy (VDE) of $0.093 \pm 0.007 \mathrm{eV}$ is in good agreement with the adiabatic dipole-bound EA computed by Adamowicz and co-workers $(0.086 \mathrm{eV}){ }^{17}$

Nearly in parallel with the work of Bowen et al., ${ }^{13}$ Schermann and co-workers reported the existence of both dipole-bound and covalent uracil anions using Rydberg electron transfer (RET) spectroscopy. ${ }^{20}$ Although the electron-attachment studies of Burrow and co-workers revealed that the vertical electron affinities of the nucleobases are all negative, ${ }^{21}$ by first forming covalent uracil-Ar anions and subsequently evaporating the solvent, Schermann et al. detected covalent uracil anions for the first time. ${ }^{20}$ However, while the RET method provides qualitative evidence for the existence of a bound covalent anion, it fails to reveal directly the magnitude of the valence electron affinity. ${ }^{20}$ Nevertheless, due to the route of anion formation, they concluded that the valence EA for uracil must be greater than the binding energies of neutral argon-uracil clusters (typically 0.030 to $0.060 \mathrm{eV}$ ), while simultaneously being smaller than the dipole-bound EA of $0.093 \mathrm{eV} .{ }^{20}$ They do concede, however, that the study of electron attachment to isolated uracil "still represents a difficult test of quantitative predictions of the valence electron affinity since its value is very close to zero." 20

Continuing in the same vein as Bowen et al., Schiedt and co-workers produced photoelectron spectra for free and microsolvated uracil as well as thymine and cytosine. ${ }^{22}$ Similarly, only dipole-bound anions were observed for the free nucleic acid bases which yield to valence-bound anions upon addition of a single water molecule. The near linear relationship between EA and the number of solvent molecules prompted these authors to estimate the EAs of the free DNA bases by extrapolation (see Figure 2). These estimates of the valence EAs and the recent work of Periquet et al. ${ }^{23}$ are the only quantitative experimentally

(20) Desfrançois, C.; Periquet, V.; Bouteiller, Y.; Schermann, J. P. J Phys. Chem. A 1998, 102, 1274.

(21) Aflatooni, K.; Gallup, G. A.; Burrow, P. D. J. Phys. Chem. 1998 , 102,6205 .

(22) Schiedt, J.; Weinkauf, R.; Neumark, D. M.; Schlag, E. W. Chem. Phys. 1998, 239, 511.

(23) Periquet, V.; Moreau, A.; Carles, S.; Schermann, J. P.; Desfrançois, C. J. Electron Spectrosc. Relat. Phenom. 2000, 141, 141. 


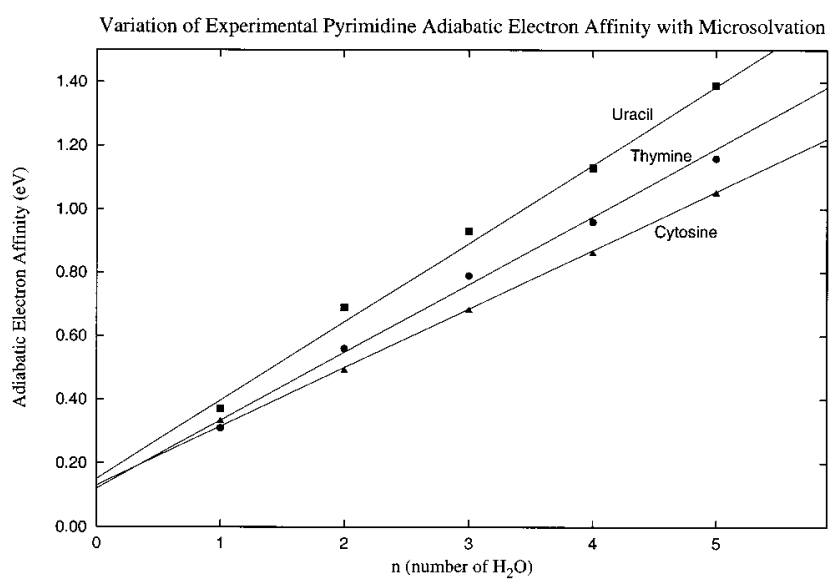

Figure 2. Schematic reproduction of the photoelectron data for the microsolvated pyrimidine nucleobase anions from ref 22 . While naked DNA base covalent anions were not observed directly, extrapolation of the $\mathrm{U}^{-} \cdot\left(\mathrm{H}_{2} \mathrm{O}\right)$ data provides an estimate of their electron affinities.

based values to complement previous studies using reduction potentials. ${ }^{15,16,24,25}$ However, the extrapolated electron affinities from the microsolvation experiments are $0.12,0.13$, and 0.15 $\mathrm{eV}$ for $\mathrm{T}, \mathrm{C}$, and $\mathrm{U}$, respectively, ${ }^{22}$ and remain far from those of Chen et al. $\left(0.79,0.56\right.$, and $0.80 \mathrm{eV}$, respectively). ${ }^{16,24,25} \mathrm{In}$ fact, the AEAs reported by Chen et al. are an average of 0.6 eV higher-in best agreement with Schiedt and co-workers' experimental EAs for the bases stablized by three water molecules. Nevertheless, the recent AM1 semiempirical multiconfiguration configuration interaction (AM1-MCCI) work of Chen and Chen bolsters their original position and they conclude that their results "...remain the only experimental and theoretically verified AEAs of adenine, guanine, cytosine, uracil, and thymine." ${ }^{25}$ Clearly, the true adiabatic electron affinities for the DNA bases are still a matter of debate.

Over the last several years, density functional theory (DFT) has emerged as an affordable, yet helpful tool for predicting electron affinities. ${ }^{26,27}$ Naturally, several studies of the nucleobase AEAs have included selected DFT computations. ${ }^{14,20,28}$ The first DFT determination of the AEA for any of the nucleobases was performed by Schermann and co-workers. ${ }^{20}$ These authors used the B3LYP functional with a $6-311 \mathrm{G}++(2 \mathrm{~d})$ basis set and predicted the AEA of uracil to be a small, but positive value $(0.070 \mathrm{eV})$. Although this AEA apparently does not include a zero-point vibrational energy correction (ZPVE), the authors note a significant nuclear rearrangement of the anion relative to the neutral. The antibonding character introduced in the covalent anion suggests that the nuclear relaxation generally would reduce the magnitude of the vibrational frequencies. Consequently, inclusion of ZPVE would likely increase the AEA of uracil. Wetmore and co-workers have recently computed AEAs for all five nucleobases using B3LYP/6-311+G(2df,p) single points at B3LYP/6-31+G(d,p) optimized geometries. ${ }^{14}$ Their predictions show thymine and uracil to have positive zeropoint corrected AEAs ( 0.14 and $0.18 \mathrm{eV}$, respectively), while cytosine, guanine, and adenine have negative AEAs $(-0.06$, -0.27 , and $-0.40 \mathrm{eV}$, respectively). Thus, the uracil and thymine covalent anions are predicted to be somewhat more

(24) Chen, E. S. D.; Chen, E. C. M.; Sane, N.; Schulze, S. Bioelectrochem. Bioenerget. 1999, 48, 69.

(25) Chen, E. C. M.; Chen, E. S. J. Phys. Chem. B 2000, 104, 7835.

(26) Galbraith, J. M.; Schaefer, H. F. J. Chem. Phys. 1996, 105, 862.

(27) Tschumper, G. S.; Schaefer, H. F. J. Chem. Phys. 1997, 107, 2529. 1243.<smiles></smiles>

Pyrimidines<smiles></smiles><smiles>Cc1ccnc(=O)[nH]c1=O</smiles><smiles></smiles>

Figure 3. Major tautomeric structures of the DNA and RNA bases. The atomic numbering scheme is identical with those in refs 14 and 28 to facilitate comparisons.

stable than the observed experimental and previously computed dipole-bound anions. 3,17,18,22,29

While B3LYP is widely considered to be one of the most reliable density functionals, systematic trends of AEAs predicted by using several density functionals have been noted over a wide range of chemical systems. ${ }^{30-35}$ In this study we predict the adiabatic electron affinities of the DNA and RNA bases using five different density functional combinations in an effort to bracket the true AEAs. In this fashion, reliable relative energy orderings of the anions are sought with an emphasis on predicting the correct sign for each nucleobase AEA. Since the signs of the EAs have remained in some doubt, a bracketing procedure which yields reliable upper bounds and plausible lower bounds to the true EAs is of great utility when dealing with values so close to zero-a range where both computations and experiments are difficult. Through this systematic approach the accuracy of the two severely conflicting sets of experimentally derived data is probed.

\section{Theoretical Methods}

Absolute energies, equilibrium structures, harmonic vibrational frequencies, and zero-point energies were determined for the neutral and anion species for each of the DNA and RNA bases shown in Figure 3. Only major tautomers are considered in this study. Five generalized gradient approximation (GGA) exchange-correlation density functionals were used and are denoted B3LYP, B3P86, BHLYP, BLYP, and BP86. These are combinations of the dynamical correlation functional of Lee, Yang, and Parr (LYP) ${ }^{36}$ or that of Perdew (P86) ${ }^{37,38}$ with one of Becke's

(29) Oyler, N. A.; Adamowicz, L. Chem. Phys. Lett. 1994, 219, 223.

(30) Rienstra-Kiracofe, J. C.; Graham, D. E.; Schaefer, H. F. Mol. Phys. 1998, 94, 767.

(31) Brinkmann, N. R.; Tschumper, G. S.; Schaefer, H. F. J. Chem. Phys. 1999, 110,6240 .

(32) Brown, S. T.; Rienstra-Kiracofe, J. C.; Schaefer, H. F. J. Phys. Chem. A 1999, 103, 4065 .

(33) Pak, C.; Xie, Y.; Van Huis, T. J.; Schaefer, H. F. J. Am. Chem. Soc. 1998, 120, 11115.

(34) Gonzales, J. M.; King, R. A.; Schaefer, H. F. J. Chem. Phys. 2000 $113,567$.

(35) King, R. A.; Pettigrew, N. D.; Schaefer, H. F. J. Chem. Phys. 1997, $107,8536$.

(36) Lee, C.; Yang, W.; Parr, R. G. Phys. Rev. B 1988, 37, 785.

(37) Perdew, J. P. Phys. Rev. B 1986, 33, 8822.

(38) Perdew, J. P. Phys. Rev. B 1986, 34, 7406. 
Table 2. Zero-Point Corrected Adiabatic Electron Affinities (in eV) of the DNA and RNA Bases with Non-ZPVE Corrected Values Given in Parentheses

\begin{tabular}{|c|c|c|c|c|c|}
\hline & uracil & thymine & cytosine & guanine & adenine \\
\hline В3P86 & $0.75(0.63)$ & $0.71(0.58)$ & $0.54(0.42)$ & $0.36(0.27)$ & $0.01(-0.08)$ \\
\hline BP86 & $0.31(0.19)$ & $0.28(0.14)$ & $0.13(0.01)$ & $0.11(0.02)$ & $-0.05(-0.15)$ \\
\hline B3LYP & $0.24(0.12)$ & $0.20(0.06)$ & $0.03(-0.09)$ & $-0.10(-0.17)$ & $-0.28(-0.37)$ \\
\hline BLYP & $0.15(0.03)$ & $0.12(-0.02)$ & $-0.01(-0.13)$ & $-0.01(-0.10)$ & $-0.19(-0.29)$ \\
\hline BHLYP & $0.06(-0.06)$ & $<0.01(-0.13)$ & $-0.14(-0.25)$ & $-0.36(-0.42)$ & $-0.65(-0.66)$ \\
\hline exptl (gas) ${ }^{a}$ & $>0^{d}$ & $>0^{d}$ & $>0 ;^{d}-0.055^{e}$ & & $-0.045^{e}$ \\
\hline exptl (scaled) $)^{b}$ & $0.80 \pm 0.05$ & $0.79 \pm 0.05$ & $0.56 \pm 0.05$ & $1.51 \pm 0.05$ & $0.95 \pm 0.05$ \\
\hline exptl (extrapolated $)^{c}$ & $0.150 \pm 0.120$ & $0.120 \pm 0.120$ & $0.130 \pm 0.120$ & & \\
\hline
\end{tabular}

${ }^{a}$ Gas-phase results. ${ }^{b}$ Scaled reduction potentials. See ref $25 .{ }^{c}$ Extrapolated values from photoelectron spectra of nucleobase $\cdot\left(\mathrm{H}_{2} \mathrm{O}\right)_{n}$ clusters. See ref 22. ${ }^{d}$ See refs 4 and $20 .{ }^{e}$ Reference 23.

exchange functionals: the 3-parameter HF/DFT hybrid exchange functional (B3), ${ }^{39}$ a modification of the half-and-half HF/DFT hybrid method as implemented in GAUSSIAN $94(\mathrm{BH}),{ }^{40}$ or the 1988 pure DFT exchange functional (B). ${ }^{41}$

Double- $\zeta$ quality basis sets with polarization and diffuse functions (denoted DZP++) were used throughout for optimizations and frequency analyses. The DZP ++ basis sets were constructed by augmenting the Huzinaga-Dunning ${ }^{42,43}$ set of contracted double- $\zeta$ Gaussian functions with one set of $p$-type polarization functions for each $\mathrm{H}$ atom and one set of five $d$-type polarization functions for each $\mathrm{C}, \mathrm{N}$, and $\mathrm{O}$ atom $\left(\alpha_{p}(\mathrm{H})=0.75, \alpha_{d}(\mathrm{C})=0.75, \alpha_{d}(\mathrm{~N})=0.80, \alpha_{d}(\mathrm{O})\right.$ $=0.85)$. To complete the DZP ++ basis, one even tempered $s$ diffuse function was added to each $\mathrm{H}$ atom while sets of even tempered $s$ and $p$ diffuse functions were centered on each heavy atom. The eventempered orbital exponents were determined according to the prescription of Lee and Schaefer: ${ }^{44}$

$$
\alpha_{\text {diffuse }} \frac{1}{2}\left(\frac{\alpha_{1}}{\alpha_{2}}+\frac{\alpha_{2}}{\alpha_{3}}\right) \alpha_{1}
$$

where $\alpha_{1}, \alpha_{2}$, and $\alpha_{3}$ are the three smallest Gaussian orbital exponents of the $s$ - or $p$-type primitive functions for a given atom $\left(\alpha_{1}<\alpha_{2}<\right.$ $\left.\alpha_{3}\right)$. The final DZP++ set contains 6 functions per $\mathrm{H}$ atom and 19 functions per $\mathrm{C}, \mathrm{N}$, or $\mathrm{O}$ atom.

In addition, single-point energies at the DZP ++ optimized geometries were computed by using a triple- $\zeta$ quality basis set (TZ2P ++ ). This basis was formed from the Huzinaga-Dunning ${ }^{45,46} s p$ sets augmented with two sets of polarization functions (two sets of five $d$-type functions on $\mathrm{C}, \mathrm{N}$, and $\mathrm{O}$, and two sets of $p$ functions on $\mathrm{H}$ ). The exponents for the polarization functions are $\alpha_{p}(\mathrm{H})=1.50,0.375$, $\alpha_{d}(\mathrm{C})=1.50,0.375, \alpha_{d}(\mathrm{~N})=1.60,0.40$, and $\alpha_{d}(\mathrm{O})=1.70,0.425$. Even tempered diffuse $s$ - and $p$-type functions were added in a fashion analogous to the $\mathrm{DZP}++$ set. The final $\mathrm{TZ} 2 \mathrm{P}++$ set contains 10 functions per $\mathrm{H}$ atom and 28 functions per $\mathrm{C}, \mathrm{N}$, or $\mathrm{O}$ atom.

Both the neutral and anion stationary points were optimized via analytic gradients until the residual root-mean-square gradient was less than $10^{-4}$ hartree/bohr. Numerical integration was performed by using the GAUSSIAN $94^{47}$ default grid consisting of 75 radial shells with 302 angular points per shell. The mass-weighted Hessian matrix, and hence the harmonic vibrational frequencies, were determined analyti-

(39) Becke, A. D. J. Chem. Phys. 93, 98, 5648.

(40) Becke, A. D. J. Chem. Phys. 1992, 98,1372

(41) Becke, A. D. Phys. Rev. A 1988, 38, 3098

(42) Huzinaga, S. J. Chem. Phys. 1965, 42, 1293.

(43) Dunning, T. H., Jr. J. Chem. Phys. 1970, 53, 2823.

(44) Lee, T. J.; Schaefer, H. F. J. Chem. Phys. 1985, 83, 1784

(45) Huzinaga, S. J. Chem. Phys. 1965, 42, 1293.

(46) Dunning, T. H. J. Chem. Phys. 1971, 55, 716.

(47) Frisch, M. J.; Trucks, G. W.; Schlegel, H. B.; Gill, P. M. W.; Johnson, B. G.; Robb, M. A.; Cheeseman, J. R.; Keith, T.; Petersson, G. A.; Montgomery, J. A.; Raghavachari, K.; Al-Laham, M. A.; Zakrzewski, V. G.; Ortiz, J. V.; Foresman, J. B.; Cioslowski, J.; Stefanov, B. B.; Nanayakkara, A.; Challacombe, M.; Peng, C. Y.; Ayala, P. Y.; Chen, W.; Wong, M. W.; Andres, J. L.; Replogle, E. S.; Gomperts, R.; Martin, R. L.; Fox, D. J.; Binkley, J. S.; Defrees, D. J.; Baker, J.; Stewart, J. P.; HeadGordon, M.; Gonzalez, C.; Pople, J. A. GAUSSIAN94, Revision C.3; Gaussian, Inc.: Pittsburgh, PA, 1995. cally for all DFT methods. All computations were carried out with the GAUSSIAN $94^{47}$ program package.

Valence adiabatic electron affinities were computed as the difference between the absolute energies of the neutral and anion species at their respective optimized geometries.

$$
A E A=E_{\text {neut }}-E_{\text {anion }}
$$

Estimates of zero-point corrected electron affinities were also determined by incorporating the appropriate harmonic ZPVE for each system.

Comparisons are made to previously reported vertical and dipolebound electron affinities. The vertical electron affinity (VEA) is defined as the difference in energy between the neutral and anion species at the equilibrium geometry of the neutral. The dipole-bound electron affinity (DBEA) refers to the energy difference between the neutral and the anion species characterized by a very diffuse molecular orbital with the additional electron weakly binding via dipole interactions rather than filling the lowest unoccupied molecular orbital as in conventional "covalent" anions. Dipole-bound states were not sought in this study given that (1) several well-established experimental and theoretical values exist, (2) the basis set would require further augmentation of extremely diffuse functions to accommodate the dipole-bound electron, and (3) DFT's ability to capture the essential dipole interactions is suspect. Thus, a complete analysis of the so-called superposition states consisting of both dipole-bound and covalent anions was not attempted. Strictly speaking, there is only a single "true" adiabatic electron affinity (i.e. the difference in energy between the most stable forms of the anion and neutral), however, the term "adiabatic electron affinity" is used throughout the text in reference to the covalent anions in a fashion consistent with previous studies.

\section{Results}

The zero-point corrected adiabatic electron affinities computed with the $\mathrm{DZP}++$ basis set are compiled in Table 2. While the electron attachment studies of Burrow and co-workers show all the vertical electron affinities of the nucleobases to be negative ${ }^{21}$ several of the functionals yield positive adiabatic electron affinities. This is consistent with the studies of Wetmore et al. $^{14}$ and Grand et al. ${ }^{28}$ and emphasizes the substantial relaxation of the nuclear framework of the anions. For example, while the neutral uracil molecule is nearly planar, its anion distorts significantly from planarity, most dramatically at the $\mathrm{C}_{4}$ carbon. ${ }^{14,20,28}$

The variation in adiabatic electron affinity with respect to choice of density functional is displayed graphically in Figures $4-6$. The AEAs of the pyrimidines are shown in Figure 4 along with the extrapolated photoelectron spectra data of Schiedt and co-workers. ${ }^{22}$ The overall variation from B3P86 to BHLYP is substantial (ca. $0.70 \mathrm{eV}$ ); however, the relative energy ordering for each nucleobase AEA is preserved across the set in a nearparallel fashion. For each pyrimidine the B3P86 prediction is $0.4 \mathrm{eV}$ greater than the next largest value (BP86), while the BHLYP results are consistently the smallest values. 


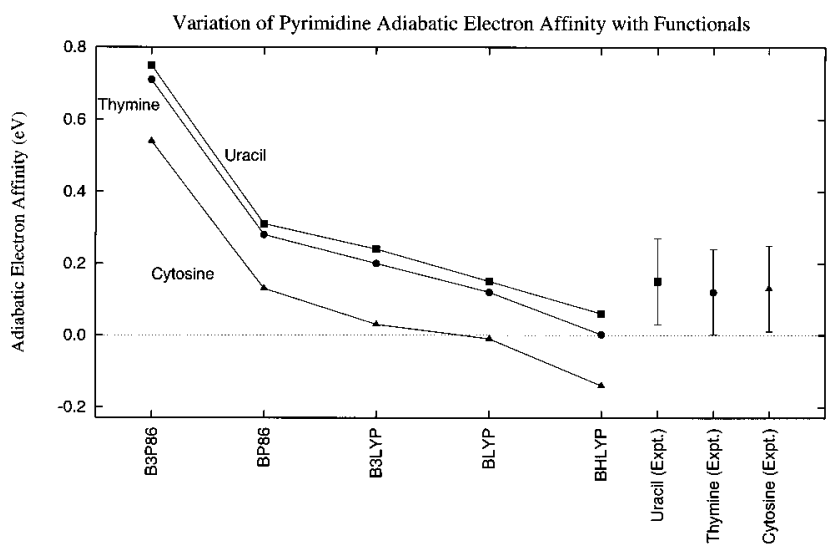

Figure 4. Variation of the adiabatic electron affinities of the pyrimidines with respect to density functional method. All computations were performed with the $\mathrm{DZP}++$ basis and are corrected for zero-point vibrational energy. Experimental values are extrapolations from photoelectron spectra of nucleobase $\cdot\left(\mathrm{H}_{2} \mathrm{O}\right)_{n}$ anion clusters (see ref 22 ).

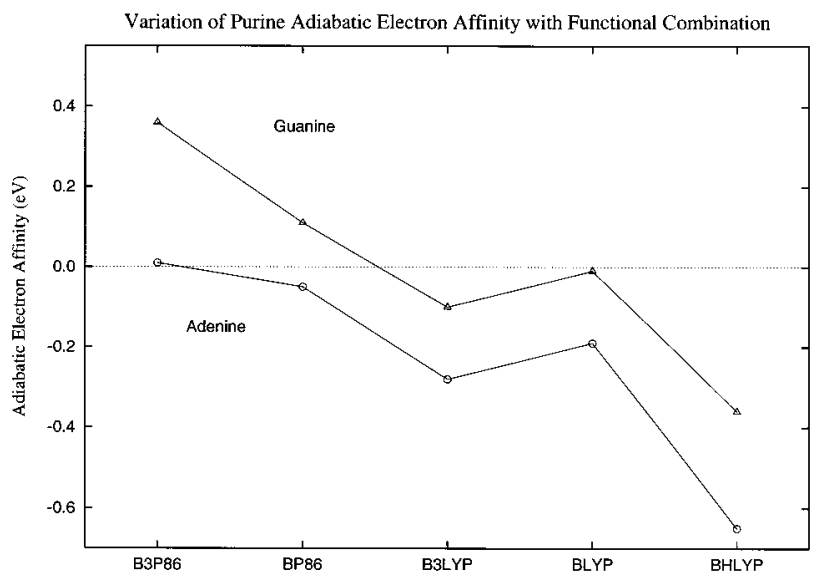

Figure 5. Variation of the adiabatic electron affinities of the purines with respect to density functional method. All computations were performed with the DZP++ basis and are corrected for zero-point vibrational energy. Note in Table 2 and in the text that the experimental values of these two electron affinities do not fall on the present diagram.

These regular trends have been well documented in a continuing series of papers ${ }^{30-35}$ and are the subject of an upcoming review. ${ }^{48}$ For example, within a set of 15 atoms and 90 molecules with known adiabatic electron affinities, the B3P86 functional with a DZP ++ basis overestimated the AEA in every case, with an average error of $0.71 \mathrm{eV}$. On the other end of the spectrum, the BHLYP functional (with its half-and-half $\mathrm{HF}$ / DFT exchange) has a noticeable tendency to underestimate AEAs (ca. 65\% of the cases studied to date). The BLYP, B3LYP, and BP86 functionals have been consistently proven to be the most accurate of the five for AEA prediction with values reliably falling between the B3P86 and BHLYP extremes. For the AEAs of uracil and thymine these three functionals are in good agreement with those determined by Schiedt et al., ${ }^{22}$ and an estimate of $0.05-0.25$ for the true AEA of each anion is reasonable. This is encouraging for the case of thymine since it has a well-established dipole-bound electron affinity of $0.07-$ 0.09 eV.22,29 Therefore, the experimental observation of the covalent thymine anion should be possible via the same RET spectroscopic procedures used to detect the covalent uracil anion. ${ }^{20}$ On the other hand, the predictions for the cytosine AEA are very close to zero (negative in some cases), and it remains unclear if its covalent anion is bound.

(48) Schaefer, H. F., et al. Chem. Rev. Manuscript in preparation.

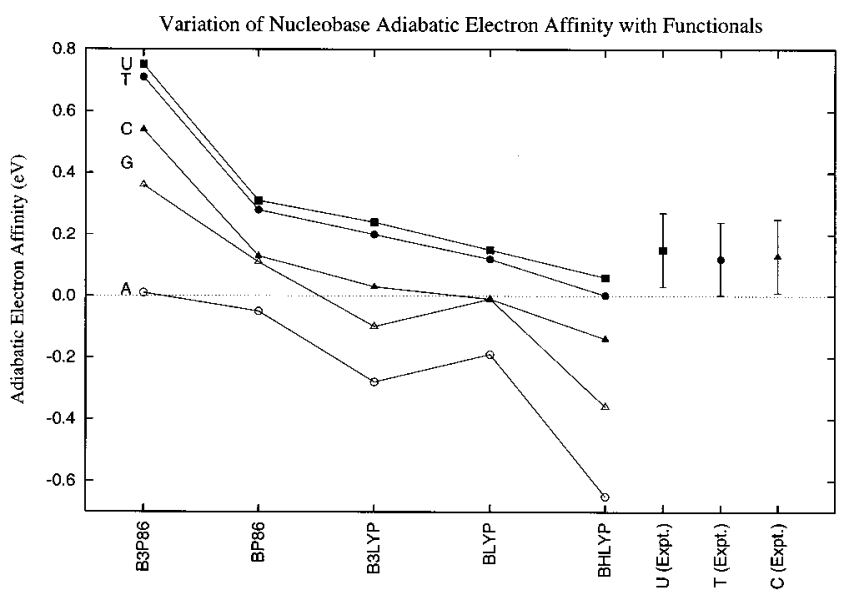

Figure 6. Variation of the adiabatic electron affinities for all DNA and RNA bases with respect to density functional. All computations were performed with the DZP++ basis and are corrected for zeropoint vibrational energy. Experimental values are extrapolations from photoelectron spectra of nucleobase $\left(\mathrm{H}_{2} \mathrm{O}\right)_{n}$ anion clusters (see ref 22). We have not attempted to place the results from the experimental liquidphase reduction potentials on this figure.

Similar trends are observed for the AEAs of the purines in Figure 5. Again the B3P86 and BHLYP values bracket those predicted with the other functionals; however, the variations are somewhat more dramatic than observed in the pyrimidines. Most notable is the substantial fluctuation (ca. $0.25 \mathrm{eV}$ ) within the three best functionals. Like cytosine, the guanine AEA oscillates between small positive and negative values and a conclusive prediction of its thermodynamic stability is not possible. On the other hand, it can be predicted with confidence that the adenine covalent anion is not stable. Since the B3P86 value $(0.01 \mathrm{eV})$ can be considered a safe upper bound to the true AEA, no experimental observation of the adenine covalent anion is expected. The results for all five nucleobases are shown on the same scale in Figure 6, and a final relative ordering of the AEAs is

$$
\mathrm{U}>\mathrm{T}>\mathrm{C} \approx \mathrm{G}>\mathrm{A}
$$

While the DFT predictions of the AEAs for $\mathrm{U}, \mathrm{T}$, and $\mathrm{C}$ are in reasonable agreement with those of Schiedt et al., ${ }^{22}$ they remain much smaller than those extracted from experimental reduction potentials by Chen and co-workers. ${ }^{16,25}$ The differences for the purines are even larger. A comparison of computed nucleobase AEAs with B3LYP/TZ2P ++ single points and both sets of experimental data is provided in Figure 7. While differences in hydrogen bonding within the nucleobase-water clusters $^{49,50}$ suggest that the linear extrapolation of photoelectron spectra of hydrated nucleobases might not be precisely valid (cytosine in particular), the deviations from the DFT results are minuscule in comparison to the reduction potential data. Chen and co-workers note that the extrapolated AEAs of Scheid and co-workers are indeed much lower than their predicted values. However, they assume: "because these values are considerably lower...they are designated as excited-state electron affinities, EA*." 25 While it is not beyond the scope of possibility that slightly more stable structural conformations for each anion may exist, ${ }^{14}$ it is unlikely that we have obtained valence excited-

(49) Mourik, T. v.; Benoit, D. M.; Price, S. L.; Clary, D. C. Phys. Chem.Chem. Phys. 2000, 2, 1281.

(50) Mourik, T. v.; Benoit, D. M.; Price, S. L.; Clary, D. C. J. Phys. Chem. A 1999, 103, 1611.

(51) Zhang, Q.; Chen, E. C. M. Biochem. Biophys. Res. Commun. 1995, 217,755 . 
Comparison of B3LYP/TZ2P++ and Extrapolated Experimental AEAs

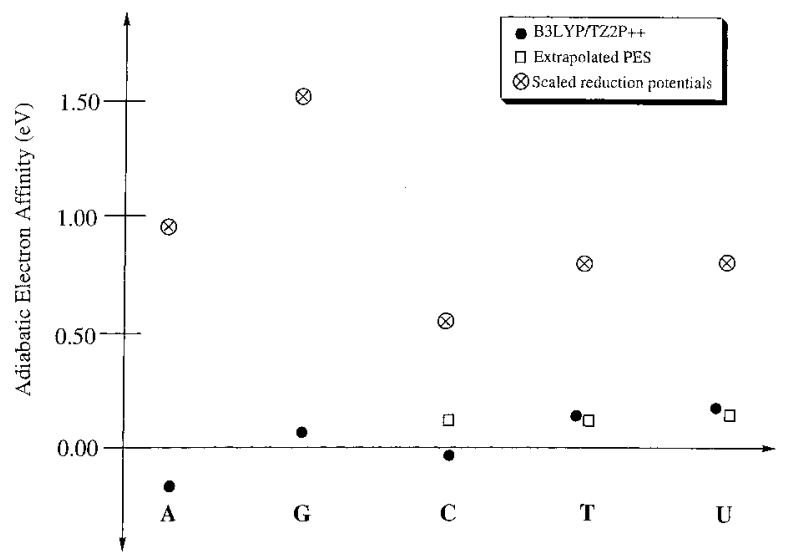

Figure 7. Comparison of $\mathrm{B} 3 \mathrm{LYP} / \mathrm{TZ} 2 \mathrm{P}++$ adiabatic electron affinities and available experimentally derived values. Single-point energies were computed at the B3LYP/DZP + optimized geometries and are corrected for zero-point vibrational energy at this same level. The ZPVE-corrected B3LYP/TZ2P++ values are $0.19(\mathrm{U}), 0.16(\mathrm{~T}),-0.02$ (C), 0.07 (G), and $-0.17 \mathrm{eV}$ (A). "Extrapolated PES" values are extrapolations from photoelectron spectra of nucleobase $\cdot\left(\mathrm{H}_{2} \mathrm{O}\right)_{n}$ anion clusters (see ref 22). "Scaled Reduction Potentials" are reversible liquidphase reduction potentials of the nucleobases which have been scaled based on known gas-phase EAs for similar compounds (see ref 16). Error bars for the experimentally derived values may be found in Table 2.

state anions given that the Kohn-Sham orbitals were constructed in $C_{1}$ symmetry. Our results (particularly the relatively small B3P86 AEAs) support the conclusions from the vertical EA studies of Burrow et al., ${ }^{21}$ who conclusively demonstrate the VEAs are slightly negative. In guanine, for example, a stabilization of almost $2 \mathrm{eV}$ by nuclear relaxation of the anion away from the geometry of the neutral would be required to account for the $1.51 \mathrm{eV}$ value of Chen et al. ${ }^{21,25}$ While we find structural relaxation to be significant (indeed, enough to yield slightly positive AEAs for uracil and thymine), we find no plausible factors accounting for a stablization of this magnitude.

\section{Conclusions}

Computed adiabatic electron affinities for the nucleobases predicted by using a range of density functionals suggest the following conclusions:
(1) The uracil (U) and thymine (T) covalent anions are bound by ca. $0.05-0.25 \mathrm{eV}$ in agreement with earlier DFT calculations by Wetmore et al. (ref 13). Furthermore, experimental AEAs for the complexes of $\mathrm{U}$ and $\mathrm{T}$ with water set firm upper bounds of ca. 0.4 and $0.3 \mathrm{eV}$ for the electron affinities of free $\mathrm{U}$ and $\mathrm{T}$, respectively. The uracil and thymine AEAs are in energy ranges near those of their dipole-bound counterparts. Therefore, experimental measurement of the thermodynamic stablility of the covalent anions is expected to be possible if they could be prepared preferentially over the dipole-bound anions.

(2) Previous theoretical and experimental estimates based on reduction potentials which place some of the AEAs for the nucleobases at $1.0 \mathrm{eV}$ or greater are clearly unreliable.

(3) Adenine does not have a stable covalently bound anion in the gas phase. This conclusion is inconsistent with experimentally based values using reduction potentials which place the electron affinity of adenine at $0.95 \pm 0.05 \mathrm{eV}$.

(4) The AEAs computed for cytosine and guanine oscillate between small positive and small negative values for the three most reliable functional combinations, and it remains unclear if either covalent anion is slightly bound or unbound. While the signs of the AEAs for thymine, uracil, and adenine given by our bracketing procedure are expected to be corroborated by eventual higher level computations, the adiabatic electron affinities of cytosine and guanine remain ambiguous.

While these conclusions further the understanding of the electronic properties and spectroscopy of the isolated nucleobases, it should be noted that this is only the first step toward a comprehensive understanding of the relevant radiation biology problems which will require treatment of base pairs, stacking, sugars, and phosphates as well as solvation.

Acknowledgment. The research conducted at the University of Georgia was supported by the U. S. National Science Foundation, grant number CHE-9827468. Sandia is a multiprogram laboratory operated by Sandia Corporation, a Lockheed Martin Company, for the United States Department of Energy under Contract DE-AC04-94AL85000. The authors wish to thank the reviewers of this manuscript as well as Nicole Brinkmann and Nancy Richardson (University of Georgia) for several insights. P.N.P. wishes to thank the members of the Center for Computational Quantum Chemistry for their hospitality and patience during his visit.

JA003814O 